\title{
ANALYTICAL FORMULAE FOR THE LOSS FACTORS AND WAKEFIELDS OF A DISK-LOADED ACCELERATING STRUCTURE
}

\author{
J. Gao \\ Laboratoire de L' Accélérateur Linéaire, IN2P3-CNRS \\ et Université de Paris-Sud,Centre d' Orsay, \\ F-91405 Orsay cedex, France
}

\begin{abstract}
In this paper we will give analytical formulae to calculate all synchronous modes' loss factors of a disk-loaded accelerating structure. From these analytical formulae one can easily find out the contribution of each synchronous mode to the total wakefields. The relations between the loss factors (wakefields) and structure geometrical dimensions have been well established. The validity of these formulae has been confirmed by using ABCI code. These analytical expressions of loss factors can be used also for a single pill-box cavity.

\section{INTRODUCTION}

Wakefields in a disk-loaded accelerating structure play a dominant role in future linear colliders. The wakefields can be calculated either in time domain by directly solving Maxwell equations, or in frequency domain by summing up synchronous modes. Usually, computer codes TBCI [1] and ABCI [2] are used in time domain, KN7C [3] and TRANSVRS [4] are used in frequency domain. In this paper, however, we give analytical expressions for the loss factors of all synchronous modes which can be used to find the delta function wakefields. These analytical expressions reveal the dependence of the loss factors on the geometric structure parameters.
\end{abstract}

\section{LOSS FACTORS OF A DISK-LOADED STRUCTURE}

A disk-loaded structure as shown in Fig. 1 can be constructed by connecting two pill-box cavities by a piece of cylindrical tube of radius $a$ and of length $d$.

Due to the coupling through the aperture between cavities, passbands will form corresponding to each pill-box resonant mode. In the following we will keep using three subscripts $m n l$ to specify the passbands without cutting the result from its origin. We assume that the synchronous frequency $\omega\left(\theta_{m n l}\right)$ is not very different from the closed pill-box resonant frequency $\omega_{m n l}$, and we will use $\omega_{m n l}$ to replace the synchronous frequency of the $\mathrm{TM}_{m n l}$ passband. In ref. 5, G. Dôme has made the same assumption to attack the same problem.

The definition of the loss factor of a synchronous mode is expressed as [6]

$$
k=\frac{E_{s, z}(r=a)^{2}}{4 d U / d z}
$$

where $E_{s, z}(r=a)$ is the synchronous decelerating electric field along the axis of $r=a$, and $d U / d z$ is the energy stored per me-

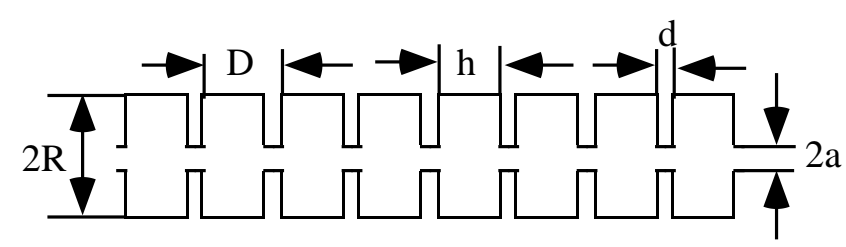

Figure 1. Disk-loaded accelerating structure

ter. For the $\mathrm{TM}_{m n l}$ passband by using the same method as in ref. 7, one has $E_{s, z}^{m n l}(r=a)=E_{z, m n l}(r=a) \eta\left(\theta_{m n l}\right)$ and $d U / d z=U_{m n l} / D$, where $E_{z, m n l}(r=a)$ is the longitudinal electric field of the $\mathrm{TM}_{m n l}$ mode in the closed pill-box cavity, and $U_{m n l}$ is the corresponding stored energy. When $\mathrm{m}=0$, $\eta\left(\theta_{m n 0}\right)$ is found to be [7]

$$
\eta\left(\theta_{m n 0}\right)=\frac{2 \sin \left(\theta_{m n 0} h / 2 D\right)}{\theta_{m n 0}}
$$

where

$$
\theta_{m n 0}=D\left(\frac{u_{m n}}{R}\right)
$$

Where $u_{m n}$ is the $n$th root of the $m$ th order Bessel function. However, if $l \neq 0$ the electric field variation inside the cavity should be taken into account. Since

$$
\cos (l \pi z / h) \cos \left(\theta_{m n l} z / D\right)=
$$

$$
\frac{1}{2} \cos \left(\left(l \pi / h+\theta_{m n l} / D\right) z\right)+\frac{1}{2} \cos \left(\left(\theta_{m n l} / D-l \pi / h\right) z\right)
$$

it is obvious that the effect of $l \neq 0$ is just splitting one synchronous mode into two and it is found by analogy that $\eta_{m n l}$ can be expressed as

$\eta\left(\theta_{m n l}\right)=\frac{\sin \left(\theta_{m n l} h / 2 D+l \pi / 2\right)}{\theta_{m n l}+l D \pi / h}+\frac{\sin \left(\theta_{m n l} h / 2 D-l \pi / 2\right)}{\theta_{m n l}-l D \pi / h}$

where

$$
\theta_{m n l}=D\left(\left(\frac{u_{m n}}{R}\right)^{2}+\left(\frac{l \pi}{h}\right)^{2}\right)^{1 / 2}
$$

We get then the general expression of the loss factor $k_{m n l}$ corresponding to the $\mathrm{TM}_{m n l}$ passband

$$
k_{m n l}=\frac{E_{s, z}^{m n l}(r=a)^{2} D}{4 U_{m n l}}
$$




$$
=\frac{\delta \xi h u_{m n}^{2} J_{m}^{2}\left(\frac{u_{m n}}{R} a\right)\left(S\left(x_{1}\right)+S\left(x_{2}\right)\right)^{2}}{\left(\left(\frac{u_{m n}}{R}\right)^{2}+\left(\frac{l \pi}{h}\right)^{2}\right) 2 \epsilon_{0} D \pi R^{4} J_{m+1}^{2}\left(u_{m n}\right)}
$$

where

$$
\begin{gathered}
\delta=\left\{\begin{array}{l}
1, l \neq 0 \\
1 / 2, l=0
\end{array}\right. \\
\xi=\left\{\begin{array}{l}
1, m \neq 0 \\
1 / 2, m=0
\end{array}\right. \\
S(x)=\frac{\sin (x)}{x}
\end{gathered}
$$

and

$$
\begin{aligned}
& x_{1}=\frac{h}{2}\left(\left(\left(\frac{u_{m n}}{R}\right)^{2}+\left(\frac{l \pi}{h}\right)^{2}\right)^{1 / 2}-\frac{l \pi}{h}\right) \\
& x_{2}=\frac{h}{2}\left(\left(\left(\frac{u_{m n}}{R}\right)^{2}+\left(\frac{l \pi}{h}\right)^{2}\right)^{1 / 2}+\frac{l \pi}{h}\right)
\end{aligned}
$$

It should be pointed out that eq. 6 is the analytical expression of the loss factors of a single pill-box cavity by setting simply $\mathrm{D}=\mathrm{h}$.

By taking all the modes into account, one can find the delta wakefield functions of a point charge traversing a disk-loaded structure by using the following formulae

$$
\begin{aligned}
& W_{z}(\tau)=\sum_{m=0}^{\infty} \sum_{n=1}^{\infty} \sum_{l=0}^{\infty} W_{z, m n l}(\tau) \\
& W_{r}(\tau)=\sum_{m=0}^{\infty} \sum_{n=1}^{\infty} \sum_{l=0}^{\infty} W_{r, m n l}(\tau) \\
& W_{\phi}(\tau)=\sum_{m=0}^{\infty} \sum_{n=1}^{\infty} \sum_{l=0}^{\infty} W_{\phi, m n l}(\tau)
\end{aligned}
$$

where [6]

$$
\begin{gathered}
W_{z, m n l}(\tau)=2 k_{m n l}\left(\frac{r r_{q}}{a^{2}}\right)^{m} \cos (m \phi) \cos \left(\omega_{m n l} \tau\right) \\
W_{r, m n l}(\tau)=\frac{2 m c k_{m n l}}{\omega_{m n l} r}\left(\frac{r r_{q}}{a^{2}}\right)^{m} \cos (m \phi) \sin \left(\omega_{m n l} \tau\right) \\
W_{\phi, m n l}(\tau)=-\frac{2 m c k_{m n l}}{\omega_{m n l} r}\left(\frac{r r_{q}}{a^{2}}\right)^{m} \cos (m \phi) \sin \left(\omega_{m n l} \tau\right)
\end{gathered}
$$

where $\tau=s / c, s$ is the distance between the exciting charge and a test charge, $r_{q}$ is the transverse coordinate of the exciting charge and $c$ is the velocity of light in vacuum. For a Gaussian bunch of charge $q$ and bunch length $\sigma_{t}$ one can calculate the integrated wakefield started from delta wakefield functions

$$
\begin{aligned}
& W_{G, z}(\tau)=\int_{-\infty}^{\tau} W_{z}(\tau-t) I(t) d t \\
& W_{G, r}(\tau)=\int_{-\infty}^{\tau} W_{r}(\tau-t) I(t) d t \\
& W_{G, \phi}(\tau)=\int_{-\infty}^{\tau} W_{\phi}(\tau-t) I(t) d t
\end{aligned}
$$

where

$$
I(t)=\frac{q}{(2 \pi)^{1 / 2} \sigma_{t}} \exp \left(-\frac{t^{2}}{2 \sigma_{t}^{2}}\right)
$$

For the $m$ th mode the total loss factor of a Gaussian bunch will be

$$
K_{m}\left(\sigma_{t}\right)=\sum_{n=1}^{\infty} \sum_{l=0}^{\infty} k_{m n l} \exp \left(-\omega_{m n l}^{2} \sigma_{t}^{2}\right)
$$

To evaluate the single bunch energy loss $\Delta U_{t}$ to all the monopole modes, one defines the beam loading enhancement factor as [6]

$$
B\left(\sigma_{t}\right)=\frac{K_{0}\left(\sigma_{t}\right)}{k_{010} \exp \left(-\omega_{010}^{2} \sigma_{t}^{2}\right)}
$$

and

$$
\Delta U_{t}=B\left(\sigma_{t}\right) \Delta U_{0}
$$

where $\Delta U_{0}$ is the energy lost to the fundamental mode.

\section{COMPARISON WITH NUMERICAL RESULTS}

In this section a special rf structure will be used to make the comparison between the calculation results from the analytical formulae and those from the ABCI code. The exciting bunch is assumed to have a Gaussian distribution and the loss factors shown in the figures are $\mathrm{k}\left(\sigma_{t}\right)$. In the following analytical calculations one has chosen $n=50$ and $l=30$ if otherwise stated. It should be declared that the width of the bars in the following pictures has no physical meanings.

We consider a disk-loaded structure as shown in Fig. 1 having the dimension: $\mathrm{D}=3.5 \mathrm{~cm}, \mathrm{~h}=2.92 \mathrm{~cm}, \mathrm{a}=1 \mathrm{~cm}$ and $25 \mathrm{~cm}$. The exciting bunch which traverses the structure parallel to the structure axis with an offset of $r=a$ has an rms bunch length $\sigma_{z}\left(\sigma_{z}=\sigma_{t} c\right)$. The analytical results of the monopole modes $(\mathrm{m}=0)$ are shown in Fig. 2 where $\sigma_{z}=2.5 \mathrm{~mm}$. Using the same structure and keeping the same exciting bunch parameters, we use $\mathrm{ABCI}$ to get the corresponding results shown in Fig. 3 where three cavities have been used. To verify the HOM mode loss factors estimated by the formulae we will compare the beam loading enhancement factor $\mathrm{B}\left(\sigma_{z}\right)$ with those calculated by ABCI. Taking the SLAC type structure parameters: $\mathrm{D}=3.5 \mathrm{~cm}, \mathrm{~h}=2.92 \mathrm{~cm}, \mathrm{a}=1 \mathrm{~cm}$ and $4.02 \mathrm{~cm}$. the beam loading enhancement factor $\mathrm{B}\left(\sigma_{z}\right)$ has been calculated, compared and shown in Fig. 4 where the fitting curve is $\mathrm{B}\left(\sigma_{z}\right)=4.66 \sigma_{z}^{-0.678}$ which agrees quite well with the diffraction model [8] which implies that the the total loss factor for a short bunch varies as $\sigma_{z}^{-1 / 2}$. For the analytical curve shown in Fig. 4, we have taken $n=200$, and $l=100$ which ensure the convergency of eq. 22 for the minimum $\sigma_{z}$ used.

The agreement between the analytical and the numerical results are obvious. The comparisons for the dipole modes have been done also, however, they are not shown in this paper due to the limited paper length.

\section{ACKNOWLEDGEMENTS}

The author thanks J. Le Duff, Y.H. Chin (KEK) and H. Braun (CERN) for the benificial communication. 


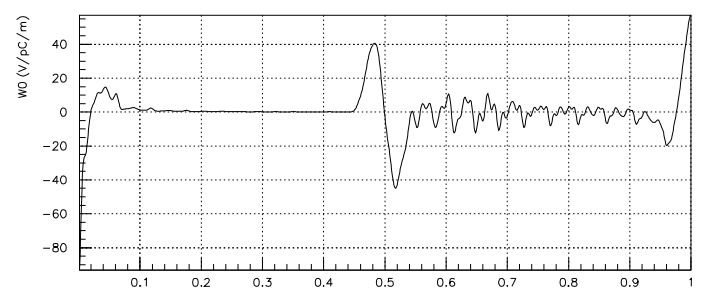

(a) $z(m)$

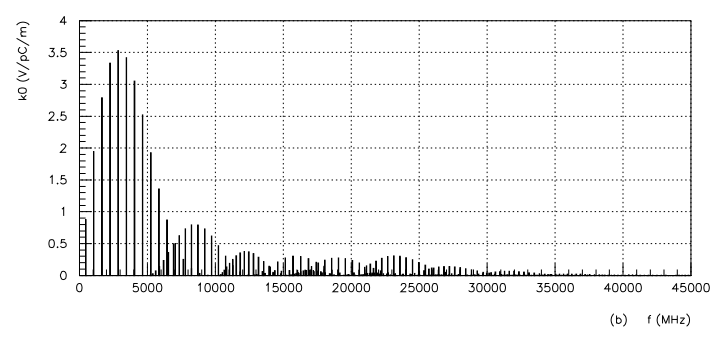

Figure 2. (a) Monopole wakefield and (b) monopole mode loss factors. For both figures $\sigma_{z}=2.5 \mathrm{~mm}$. The dimension of the structure: $\mathrm{D}=3.5 \mathrm{~cm}, \mathrm{~h}=2.92 \mathrm{~cm}, \mathrm{a}=1 \mathrm{~cm}$ and $\mathrm{R}=25 \mathrm{~cm}$. (Formulae)

\section{References}

[1] T. Weiland, DESY 82-015, 1982.

[2] Y.H. Chin, "User's guide for ABCI version 8.8", LBL35258.

[3] E. Keil, "Diffraction radiation of charged rings moving in a corrugated cylindrical pipe", Nucl. Instr. and Meth., A100 (1972) 419.

[4] K. Bane and B. Zotter, Proc. of the 11th International Conference of High Energy Accelerators, Geneva (1980) p. 581.

[5] G. Dôme, "Wake potentials of a relativistic point charge crossing a beam-pipe gap: an analytical approximation", IEEE Trans. on Nucl. Science, NS-32 (1985) 2531.

[6] P.B. Wilson, "Introduction to wake potentials", SLACPUB-4547 (1989).

[7] J. Gao, "Analytical approach and the scaling laws in the design of disk-loaded accelerating structures", Particle Acclerators, 43 (1994) 235.

[8] K. Bane and M. Sands, "Wakefields of very short bunches in an accelerating cavity", SLAC-PUB-4441 (1987).

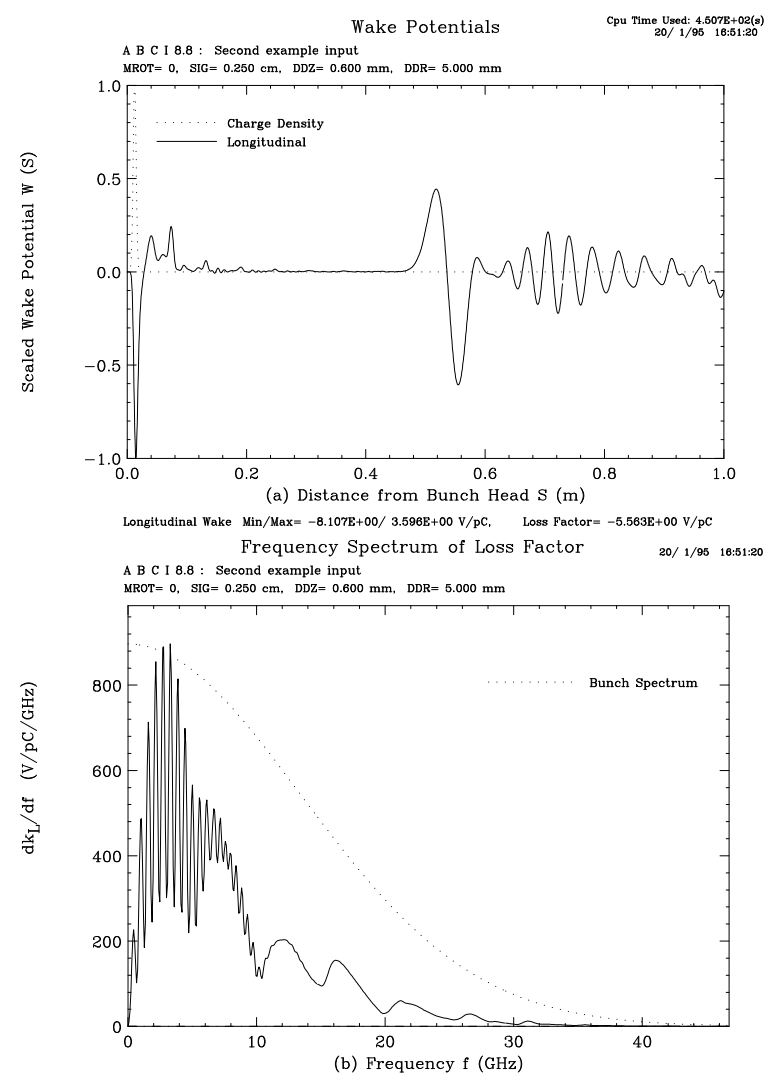

Figure 3. (a) Monopole wakefield and (b) Monopole mode frequency spectrum of loss factors. For both figures $\sigma_{z}=2.5 \mathrm{~mm}$. The dimension of the structure: $\mathrm{D}=3.5 \mathrm{~cm}, \mathrm{~h}=2.92 \mathrm{~cm}, \mathrm{a}=1 \mathrm{~cm}$ and $\mathrm{R}=25 \mathrm{~cm}$. $(\mathrm{ABCI})$

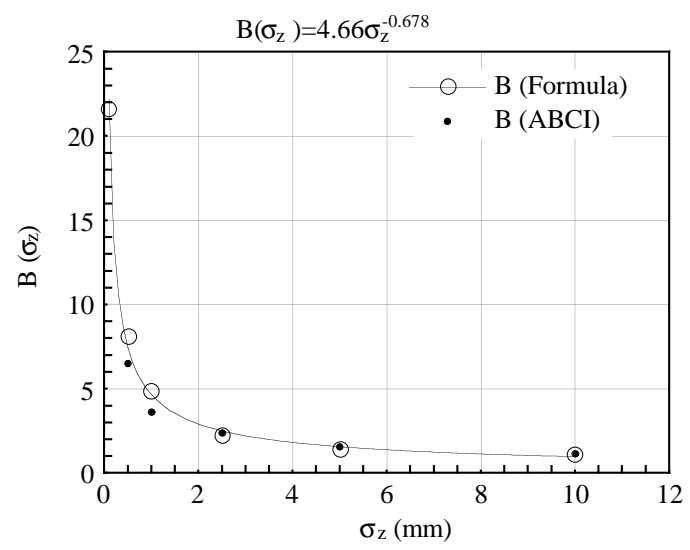

Figure 4. The comparison of the enhancement factors $\mathrm{B}\left(\sigma_{z}\right)$ calculated by the analytical formula and ABCI code for a SLAC type structure. The dimension of this structure: $\mathrm{D}=3.5 \mathrm{~cm}$, $\mathrm{h}=2.92 \mathrm{~cm}, \mathrm{a}=1 \mathrm{~cm}$ and $\mathrm{R}=4.02 \mathrm{~cm}$. 